\title{
Laser-Induced Backside Wet Etching of Transparent Materials with Organic and Metallic Absorbers
}

\author{
K. Zimmer and R. Böhme \\ Leibniz-Institut für Oberflächenmodifizierung, Permoserstraße 15, 04318 Leipzig, Germany \\ Correspondence should be addressed to K. Zimmer, klaus.zimmer@iom-leipzig.de
}

Received 24 July 2008; Accepted 27 August 2008

Recommended by Jacques Albert

\begin{abstract}
Laser-induced backside wet etching (LIBWE) allows the high-quality etching of transparent materials for micro- and nanopatterning. Recent own results of LIBWE with hydrocarbon and metallic absorbers (H- and M-LIBWE) are summarized and compared with selected results of other groups regarding the etching process and the etched surface. Significant results on the impact of the liquid absorber, the material and the wavelength, and the pulse length of the laser to the etching are selected for this comparison. The etching of submicron-sized periodic structures in sapphire and fused silica with interference techniques and the selection of the preferred method in dependence on the material and the processing goal discussed. The experimental results are discussed on a thermal model considering both interface and volume absorption of the laser beam. These results have the conclusion that the etching at M-LIBWE is mainly due to material melting and evaporation whereas at H-LIBWE, a modified near-surface region with a very high absorption is ablated.
\end{abstract}

Copyright ( $\odot 2008$ K. Zimmer and R. Böhme. This is an open access article distributed under the Creative Commons Attribution License, which permits unrestricted use, distribution, and reproduction in any medium, provided the original work is properly cited.

\section{Introduction}

The high-quality, precise, low-damage processing of transparent dielectrics by lasers with respect to high-quality micro- and nanopatterning of these materials is still a challenge [1-3]. Therefore, the lateral and vertical resolution of the patterning process should be reduced and the surface quality, that is roughness, waviness, and processing-induced near-surface material modification, must be improved.

In addition to traditionally used nanosecond lasers that allow ablation of such materials, for example, fused silica, at high laser fluences $[4,5]$, nowadays ultrashort pulse or VUV lasers are exploited increasingly [6-9]. These lasers cause material ablation after multiphoton and singlephoton excitation and enable material processing at low laser fluences with lower ablation rates which can result in improved surface qualities. In consequence, one key for high-quality materials processing is a high absorption of the laser beam by the material, in particular the surface. This results in near-surface energy deposition, high energy densities, and fast phase transitions and prevents excessive melting of the material already at low laser fluences.

With the aim of absorption enhancement of transparent materials, two beam laser ablation techniques have been developed. Principally, a first low-fluence high-photonenergy laser is used for the generation of free electrons and temporary defects whereas a second high-fluence (UV) laser is employed for ablation after photon absorption by the laser-induced transient absorption centres $[10,11]$. Such temporary modifications for surface absorption enhancement were applied also to backside ablation. Zhang etal. introduced laser-induced plasma-assisted ablation (LIPAA) that makes use of laser-induced plasma from metal plate placed behind the transparent sample. The laser plasma modifies the backside of the transparent sample and the enhanced laser absorption resulting in backside ablation [12$16]$.

Nowadays a number of indirect laser processing methods is in development that aims also to a near-surface absorption of the laser energy at the backside of transparent samples. However, the enhanced surface/interface absorption is 
TABLE 1: Main characteristics of the backside etching techniques with nanosecond laser pulses in comparison to laser ablation.

\begin{tabular}{lccc}
\hline Characteristic & & Technique & \\
& H-LIBWE & M-LIBWE & Ablation \\
\hline Threshold fluence & Low & High & High \\
Laser wavelength & UV/vis & UV-IR & UV (IR) \\
Etch rate & Average & High & High \\
Incubation effects & Distinct & No & Noticeable \\
Etching quality & Very good & Good & Average \\
\hline
\end{tabular}

achieved at these methods by additional materials. Depending on the additive to enhance the energy deposition near the transparent solid surface, different techniques can be distinguished.

The laser etching by surface-adsorbed layer (LESAL) technique uses hydrocarbon layers adsorbed at the backside of the sample to enhance the laser beam absorption [17-20]. Recently, the so-called laser-induced backside dry etching (LIBDE) was demonstrated by Hopp etal. using a thin metal film for laser energy deposition [21]. Laser-induced backside wet etching (LIBWE) developed by Niino's group makes use of the high absorption of organic liquids (H-LIBWEhydrocarbon LIBWE) for excimer laser wavelengths [2224]. Previously Shafeev performed material processing at the interface to liquids with a similar set-up but uses much higher laser fluences of a high repetition rate copper vapour laser [25, 26]. Zimmer et al. applied liquid metals (M-LIBWE-liquid metal LIBWE) instead of the initially exploited organic solutions for backside wet etching [27-29].

To ensure a sufficiently high absorption of the organic liquids, UV excimer lasers ( $\mathrm{XeF}, \mathrm{XeCl}, \mathrm{KrF}, \mathrm{ArF}$ ) have to be used for H-LIBWE. Higher harmonics of high repetition rate Nd:YAG lasers $(530 \mathrm{~nm}, 266 \mathrm{~nm})$ were applied for etching, too $[30,31]$. However, the utilization of visible wavelengths requires special dyes for absorption and in addition higher laser fluences [30]. For very high repetition rates, the bubble lifetime can exceed the pulse interval whereby the etching process is influenced and lower etch rates were found [30, 31]. A wide range of liquids were investigated for H-LIBWE comprising mainly solutions of dyes in organic solvents $[22,32]$, solutions of inorganic and organic absorbers in water [25, 26, 33, 34], and liquid metals [27, 35]. Only some experiments on LIBWE with ultrashort pulse lasers are known $[36,37]$.

The most studies aim at the effects of specific material processing conditions (liquid, wavelength, etc.) on the etching process and the properties of the etched surface. The effect of H-LIBWE to the properties of the processed surface was investigated in $[38,39]$ and gave evidence for a strong surface modification and incubation. Some studies aim at the comparison of experimental LIBWE results with (numerical) model calculations [32, 40]. In [32], it was found that the interface temperature at H-LIBWE is below the melting temperature of the fused silica for the laser fluences at the etch threshold; however, in this work interface modifications were neglected. In Table 1, the

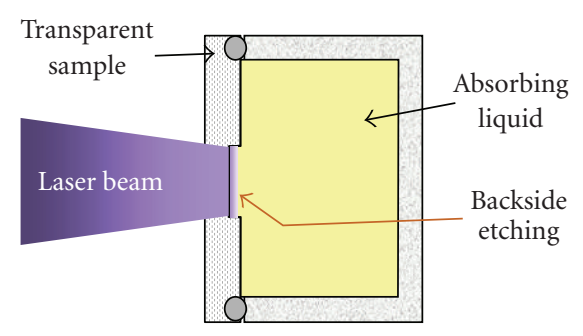

FIgURE 1: Basic experimental set-up for LIBWE.

most important characteristics of patterning processes for transparent materials are listed for $\mathrm{H}$ - and M-LIBWE in comparison to laser ablation.

All the mentioned backside etching techniques have been used for micro- and nanopatterning of different transparent glass and crystals [22, 23, 41-43]. Examples are the etching of submicron and nanometer gratings in fused silica [4446] and sapphire [47], the submicron patterning on-thefly with one laser pulse [48], the etching of micro-optical elements $[18,39,42,49-53]$, and the fabrication of arrays for biomedical purposes $[30,54]$.

\section{Experimental}

The basic configuration of the experimental set-up for LIBWE is shown in Figure 1. The etching chamber holds the substrates and contains the liquid absorber. The chamber assembly is often incorporated into a laser workstation or a similar installation for precise processing and full parameter control as has been described elsewhere $[30,55]$. In summary, the laser beam goes trough the transparent sample and is absorbed at its backside by the liquid.

Mainly the following laser parameters were varied with one set-up: the spot size, the laser fluence, the pulse number. Depending on the laser source and the beam quality, the laser beam is directly focussed or used for mask projection. In consequence, the intensity distribution of the laser spot is either Gaussian-like for solid state lasers (e.g., Nd:YAG) or uniform with an average deviation in the range of $\pm 5 \%$ for excimer lasers. The wavelength, the pulse length, and the temporal pulse shape are also characteristics of the used laser source: nanosecond (UV: XeF, KrF, ArF excimer lasers and IR: Nd:YAG laser; $t p \sim 20, \ldots, 30$ nanoseconds) as well as picosecond laser pulses (4th Nd:YAG laser, $t p=$ 150 picoseconds) were applied. Usually low repetition rates $(<10 \mathrm{~Hz})$ were used in the basic experiments. To achieve high spatial resolution down to the submicron range, interference techniques such as phase mask projection [44] and interferometer set-ups [47] were applied.

\subsection{Samples}

For backside etching, double-side polished high-quality substrates were used. The material of choice in most of the experiments was optical-grade fused silica (FS). Further, 
crystalline materials such as quartz, sapphire, and fluorides $\left(\mathrm{CaF}_{2}, \mathrm{MgF}_{2}\right)$ with a thickness of some mm were utilized. To allow high-resolution mask projection, thin samples $(<1 \mathrm{~mm})$ were used. The surface roughness was always in the range of $1 \mathrm{~nm}$ rms or even lower; the fused silica samples regularly have a roughness of about 0.2 to $0.3 \mathrm{~nm}$ rms. The samples were used as received without further cleaning.

\subsection{Liquids}

Two classes of liquids are used: (i) hydrocarbon liquids that are solutions of pyrene in toluene (py:toluene), acetone, tetrachloroethylene, and so forth, at concentrations of usually $0.5 \mathrm{M}(\mathrm{M}=\mathrm{mol} / \mathrm{L})$ and (ii) liquid metals such as gallium $(\mathrm{Ga})$ and mercury $(\mathrm{Hg})$.

\subsection{Processing Procedure}

After mounting the samples to the chamber, the etching was performed. For large area processing, for example, writing of arrays or micro-optical patterns, a circulating pump assembly might be used [30]. This allows the continuous exhaust, the filtering, and the cooling down of the used solution. After processing the samples were cleaned from the liquid absorber either by washing in organic solvents (acetone, ethanol, etc.) or by etching in suitable acids (e.g., nitric acid); ultrasonic can be applied, in addition. Subsequently, the samples were washed in water and dried in a dry nitrogen stream. For removing adherent carbon contaminations a soft oxygen-plasma cleaning procedure was applied, too [37].

\subsection{Analytic of Etched Surfaces}

The topography over the full range of spatial frequencies, laser-induced modifications, and the near-surface material properties (composition, absorption structure, etc.) of the etched samples were investigated by the following analytical techniques: Scanning electron microscope (SEM, either a JEOL JSM 6600 or a Zeiss CARL ZEISS Ultra 55) was employed for taking top-view or cross-section images of the etched samples. White-light interference microscopy (e.g., Micromap 512) was frequently performed to measure the etch depth, the topography, the waviness, and the roughness ( $\mu \mathrm{m}$-range) of the etched surfaces with an accuracy down to $1 \mathrm{~nm}$ in depth. High-resolution investigations of the topography and the roughness are performed by AFM measurements using a Nanoscop III instrument. The transmission of irradiated sample regions was measured utilizing a scanning UV/VIS spectrometer (Shimadzu UV-2101PC UV/VIS scanning spectrophotometer, $\lambda=200-800 \mathrm{~nm}$ ). For determining structural defects in solid material and nearsurface contaminations channeling/RBS- (Rutherford Back Scattering) measurements with a $2 \mathrm{MeV}-\mathrm{He}^{2+}$ beam of about $1.5 \mathrm{~mm}$ diameter were performed.

\section{Experimental Results for Laser Etching of Transparent Materials}

As already mentioned the most principal task of the rear side liquid is the absorption of the laser radiation near the solid backside surface. Therefore can be expected a better the material etching for laser beam absorption closer to the surface. Two basic classes of liquid absorbers must be considered:

(i) hydrocarbon liquids or solutions (H-LIBWE),

(ii) liquid metals (M-LIBWE).

The classical H-LIBWE requires nanosecond-pulsed UV or VIS laser to match the absorption of the liquid. Nevertheless, typical laser penetration depths of organic liquids are some microns. The liquid metal LIBWE permits the utilization of IR laser pulses for etching since metal absorbs efficiently over the whole range of the spectrum with a short penetration depths $\left(\alpha^{-1} \ll 100 \mathrm{~nm}\right)$.

Typical images of the etched fused silica surfaces are shown in Figure 2 for H-LIBWE with py:toluene in (a) and M-LIBWE with gallium in (b). The size and the shape of the edges and the etched bottom show a very good quality in both cases. Although the microroughness is low for both techniques, M-LIBWE shows a higher waviness of the etched surface that looks like frozen waves. These features together with the high etch rates let us suggest that the material etching for M-LIBWE involves a molten phase that resolidifies after material ejection.

\subsection{Etch Rate of Fused Silica}

The most accessible and informative experimental result is the etch rate of the material depending on the laser fluence and the pulse number. The etch rate of fused silica for $248 \mathrm{~nm}$ excimer laser pulses depending on the laser fluence and the pulses number is shown in comparison for H-LIBWE with a $0.5 \mathrm{~mol} / \mathrm{L}$ pyrene/toluene solution $(0.5 \mathrm{M}$ py:toluene) and M-LIBWE with gallium in Figure 3, respectively.

At least three etching characteristics are different for both approaches: (i) M-LIBWE offers much higher etch rates than H-LIBWE but (ii) requires much higher laser threshold fluences for etching. In addition, (iii) strong incubation effects were detected at H-LIBWE whereas M-LIBWE features almost no incubation effects $[27,56]$. The higher threshold fluences at M-LIBWE can be explained in parts by the higher reflectivity of the interface and the higher thermal conductivity of the liquid metal absorber, respectively [28, 35]. Because all other experimental parameters are similar, the incubation must be linked to the used liquid that should cause an additional laser irradiation-induced change of the interface region in order to enhance the laser absorption either in the liquid or in the near-solid region. The negligible incubation effect at M-LIBWE can be explained by the high absorption coefficient and the high stability of the liquid metals. On the other hand, the organic liquids and solutions used at H-LIBWE suffer from photothermal and/or 


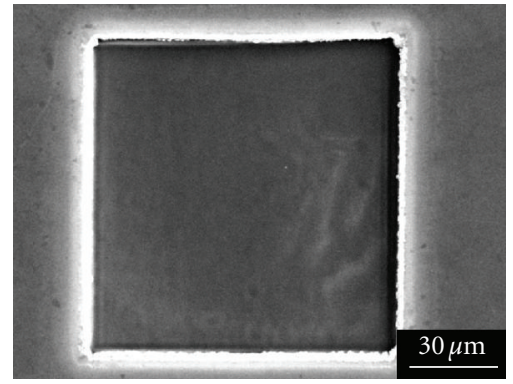

(a)

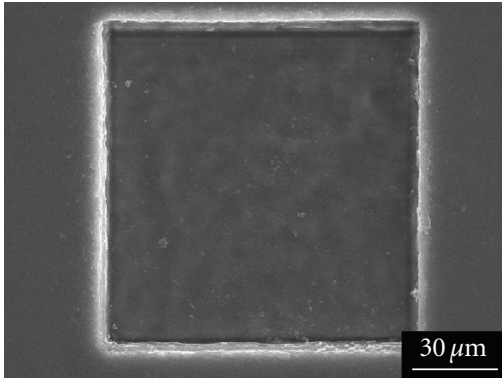

(b)

FIGURE 2: SEM images of etched square patterns in fused silica with $100 \times 100 \mu \mathrm{m}^{2}$ by $248 \mathrm{~nm}$ excimer laser: (a) H-LIBWE with py:toluene (300 pulses at $\left.1.4 \mathrm{~J} / \mathrm{cm}^{2}\right)$ and (b) M-LIBWE with $\mathrm{Ga}\left(30\right.$ pulses at $\left.3.4 \mathrm{~J} / \mathrm{cm}^{2}\right)$.

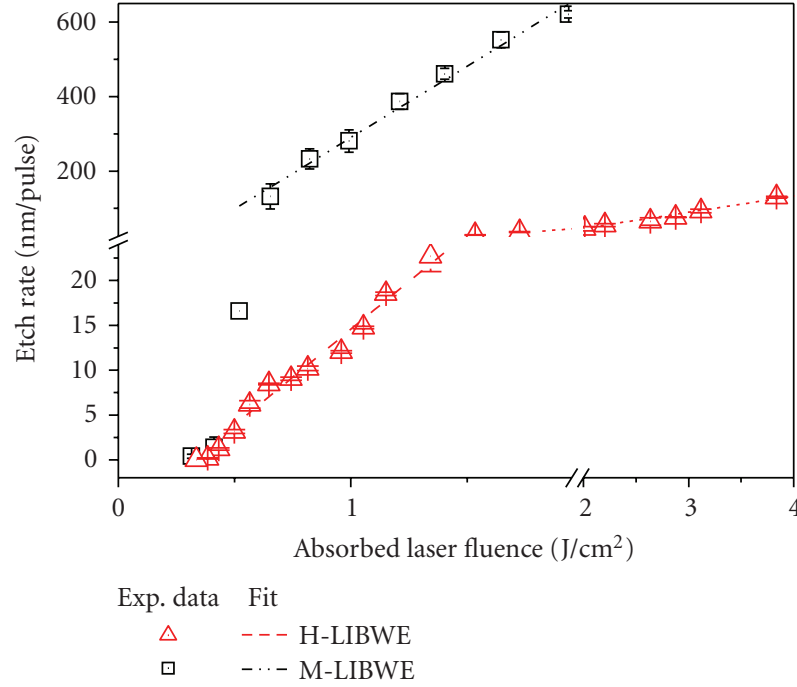

(a)

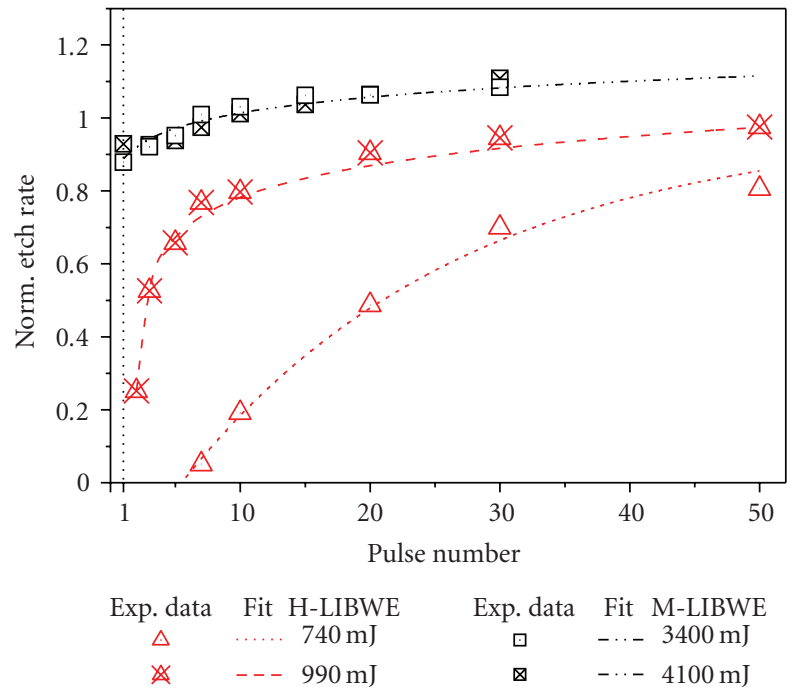

(b)

FIGURE 3: Etch rate of fused silica with $248 \mathrm{~nm}$ excimer laser pulses and a spot with size of $100 \times 100 \mu \mathrm{m}^{2}$. Comparison of H-LIBWE with a $0.5 \mathrm{M}$ py:toluene and M-LIBWE with gallium. (a) Etch rate depending on the laser fluence. (b) Normalized etch rate depending on the pulse number.

photochemical decomposition under intense pulsed UV laser irradiation [57].

The analysis of hydrocarbon solutions showed among a lot of soot a number of decomposition products at HPLCMS due to laser exposure. However, the composition of the liquid is not altered in this extent to explain a much higher (local) absorption coefficient of the liquid [58]. The decomposition of hydrocarbon liquids under UV laser irradiation can result in conjunction with high temperatures to the formation of excited atoms or radicals. This can be of interest when halogenated hydrocarbons are used that might form chemical radicals that are able to etch solid materials directly [59].

\subsection{Etch Characteristics for Different Materials and Absorbing Liquids}

The etch characteristics, for example etch rate and surface morphology, depend on the used liquid for both classes of liquid absorbers: hydrocarbon solutions and liquid metals. The etch rates of fused silica are shown in Figure 4 for two different absorbers in each case-gallium and mercury and py:toluene and py:halogenated benzenes. In both cases, the threshold fluence and the etch rate dependency are affected by the used liquid. The comparison for M-LIBWE in Figure 4(b) shows that mercury has a less etch threshold (Hg: $0.76 \mathrm{~J} / \mathrm{cm}^{2}$; Ga: $1.3 / 2.1 \mathrm{~J} / \mathrm{cm}^{2}$ ) and a slightly lower etch rate than gallium. However, in both cases a linear rise of the etch rate, no incubation effects, and similar etch pits with well-defined edges and flat bottoms have been observed $[27,29]$. The lower threshold of mercury can be explained by the twofold lower thermal diffusion length (Hg: $0.66 \mu \mathrm{m}$; Ga: $1.24 \mu \mathrm{m}$ at $t p=25$ nanoseconds). The lower slope of the etch rate raise for mercury can be discussed also by the different material properties of mercury.

The etch rate dependencies achieved for H-LIBWE of fused silica using either pyrene-doped toluene or monohalogenated benzenes $(0.5 \mathrm{M})$ are rather dissimilar; however, 


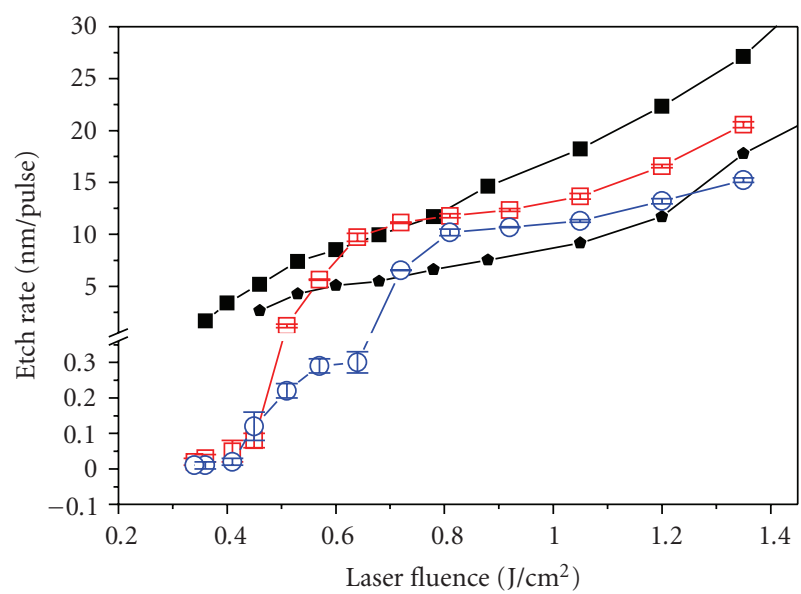

Pyrene concentration

$0.5 \mathrm{M} \quad 0 \mathrm{M}$

$-\quad-\square-$ Toluene

$-\square-\quad \mathrm{C}_{6} \mathrm{H}_{5} \mathrm{Cl}$

$-\odot-\quad \mathrm{C}_{6} \mathrm{H}_{5} \mathrm{~F}$

(a) H-LIBWE

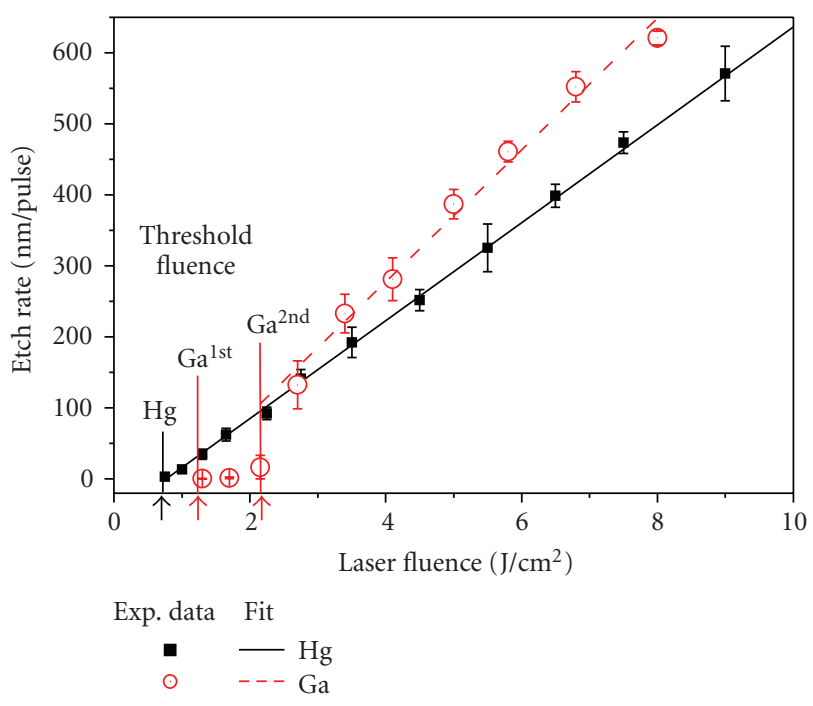

(b) M-LIBWE

FIGURE 4: Etch rates of fused silica for different liquids at $248 \mathrm{~nm}$ laser pulse irradiation. (a) Etch rate of fused silica for toluene with and without solved pyrene and for halogenated benzenes (fluorine and chlorine benzene) at 300 pulses. (b) Etch rate for fused silica at 30 pulses using gallium and mercury as liquid metals.

above a certain laser fluence of about $0.7 \mathrm{~J} / \mathrm{cm}^{2}$, similar etch rates and a linear rise can be observed. Especially at low fluences, a much lower rate and a higher slope of the rate were measured for the halogenated solvent although the absorption coefficient was similar due to the high pyrene concentration. It should be noted that the threshold fluence for the pyrene-doped solutions is similar $\left(\sim 0.33 \mathrm{~J} / \mathrm{cm}^{2}\right)$ but significantly less than the threshold for pure toluene $\left(0.45 \mathrm{~J} / \mathrm{cm}^{2}\right)$. The etch rate with pure toluene also increases almost linearly but is only a bit less than that achievable with pyrene. Though the threshold depends on the liquid absorption, there is no linear correlation as the pyrene doping causes a sevenfold increase of the absorption but the threshold is reduced only little.

The laser-induced decomposition of halogenated hydrocarbons, however, can result in halogen radicals $[57,58,60,61]$ and can, therefore, contribute to the etching $[58,62]$. However, this does not explain the lower etch rate if at halogenated organics the same processes occur. Therefore, the halogen radicals may also affect the overall processes of the etching, for example, in the liquid, vapour, bubble, and so forth, where different chemical reactions and physical processes might occur.

Similar to the liquid absorber, the properties of the material etched by LIBWE affect the etch result. The SEM images in Figure 5 depicting etch pits in sapphire, calcium fluoride $\left(\mathrm{CaF}_{2}\right)$, and fused silica show this clearly. In contrast to the flat bottom and the well-defined edges of the fused silica, the etched patterns of both sapphire and $\mathrm{CaF}_{2}$ feature an uneven, grainy surface and an irregular edge.

The main differences of the materials that can account for the morphological changes are the thermal properties and the mechanical and chemical resistance of the materials. For instance, the thermal conductivity $k$ of the crystals (sapphire: 40 and $\left.\mathrm{CaF}_{2}: 9.7 \mathrm{~W} \cdot(\mathrm{m} \cdot \mathrm{K})^{-1}\right)$ is much higher than that of fused silica $\left(1.4 \mathrm{~W} \cdot(\mathrm{m} \cdot \mathrm{K})^{-1}\right)$. Therefore, the higher heat diffusion length forces the heat dissipation and does not allow as high heating rates and high temperatures as for fused silica. With these arguments, the surface morphology of sapphire and $\mathrm{CaF}_{2}$ can be interpreted by melting, melt ejection, and resolidification of a near-surface layer. The formation of microcracks in these brittle materials must be taken into account in addition.

\subsection{Modification of the Etched Surface at H-LIBWE}

The surfaces of etched fused silica and quartz samples were investigated with different analytical techniques such as XPS, Raman spectroscopy, and RBS to study the process-induced near-surface chemical and structural modifications [23, 39, $62,63]$. The kind and the amount of the modification depend on the applied laser fluence and the pulse number [58]. In general, with increasing laser fluence, the mainly found carbon contaminations at low fluences reduce whereas the depth of structural transformations increases.

To study the impact of the near-surface material modifications on the etching process, optical transmission measurements were performed. The inset of Figure 6 shows the transmission of an etched fused silica sample $\left(0.76 \mathrm{~J} / \mathrm{ccm}^{2}\right)$ depending on the wavelength $(200 \mathrm{~nm}$ to $800 \mathrm{~nm}$ ). In general, a reduced transmission in the UV is obvious and can also be observed for other fluences and pulse numbers. 


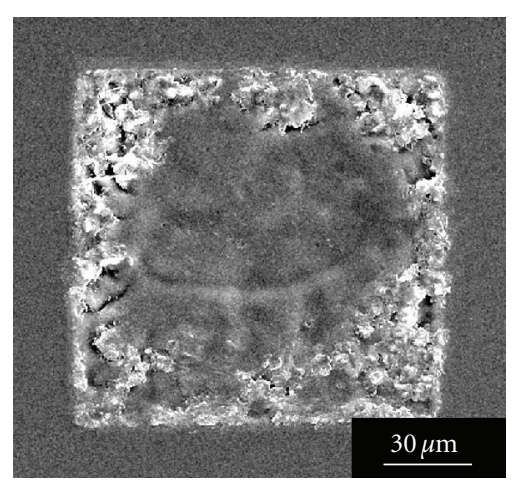

(a)

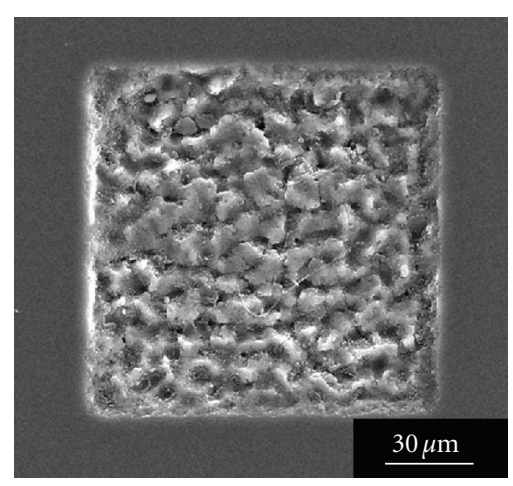

(b)

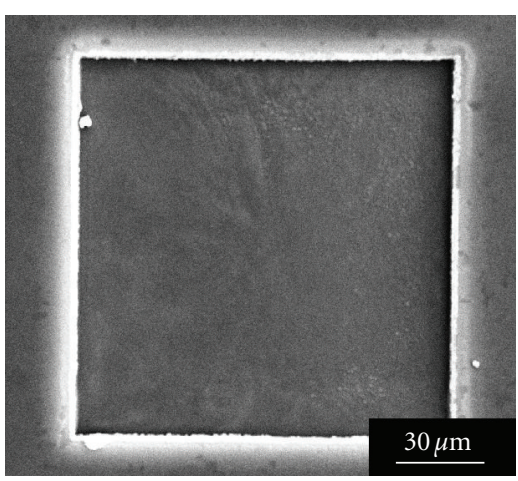

(c)

Figure 5: Surface morphology of H-LIBWE etched sapphire (a) and $\mathrm{CaF}_{2}(\mathrm{~b})$ in comparison to the standard material fused silica (c).

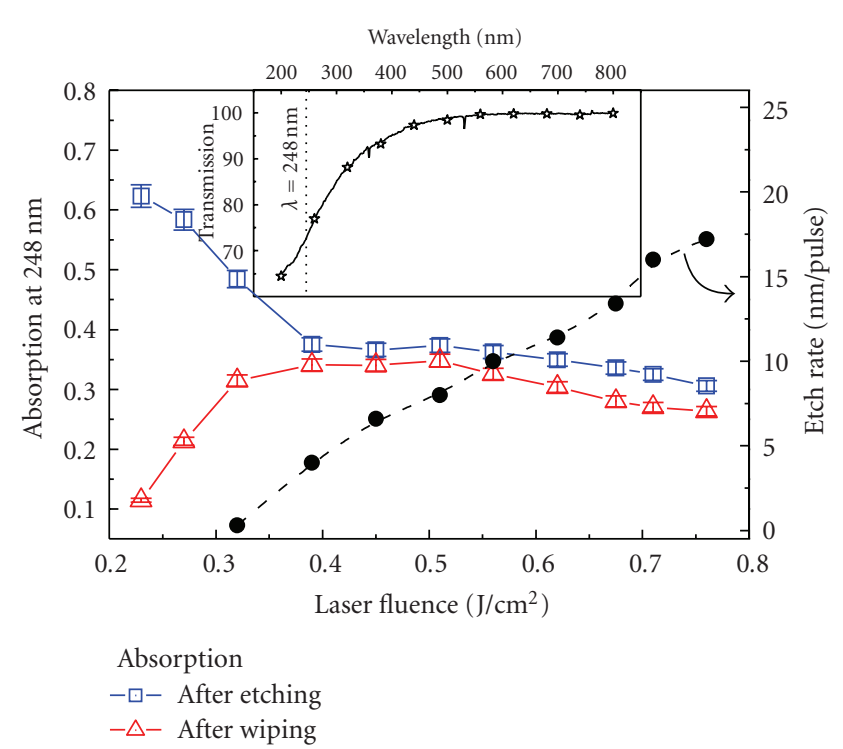

Figure 6: Absorption of etched fused silica surface depending on the laser fluence. The inset shows spectra of an etched fused silica sample $\left(\mathrm{F}=0.76 \mathrm{~J} / \mathrm{cm}^{2}\right)$ with a reduced transmission in the UV.

According to [58], the spectrum can be interpreted as a superposition of the spectra from carbon and defectenriched fused silica. Thus, the observed changes of the spectra taken at different etch conditions also refer to alterations of the surface modification. The change of the absorption at $\lambda=248 \mathrm{~nm}$ with the applied laser fluence for etched and wiped samples is shown in Figure 6, too. It should be noted that surface modifications occur below the threshold and the modifications can be divided in loosely adherentthat can be whipped away-and incorporated modifications. Together with the XPS and Raman spectroscopy results, the loosely adherent modifications are mainly carbon or carbon products from the decomposition of the organic absorber [58]. The near-surface fused silica modifications can be removed completely by ion beam sputtering of about $50 \mathrm{~nm}$ $[58,64]$. This depth is similar to the depth of structural defects found in quartz [63].
The remarkable high absorption of a thin $(<50 \mathrm{~nm})$ nearsurface region of about 30 to $40 \%$ is due to structural modifications of the fused silica and a chemical modification of the surface or by a carbon-containing film.

\subsection{Etching of Submicron Patterns in Transparent Materials}

From the basic results, it is clear that micropatterning of fused silica can be easily achieved with different liquids and laser sources. A lot of examples are known for different technical fields. However, with the reduction of the feature size, the requirements for the etching process are enhanced concerning the resolution of the etching process, the homogeneity of the etching, and the smoothness of the surface.

\subsubsection{Submicron Grating Etching in Fused Silica}

These requirements for etching of submicron patterns are fulfilled for fused silica for both $\mathrm{H}$ - and M-LIBWE. The first experiments on etching high-resolution $1 \mathrm{D}$ gratings with a period of $\sim 750 \mathrm{~nm}$ are presented in [44]. Depending on the average fluence and the pulse number, grating depths of up to $180 \mathrm{~nm}$ with a nearly sinusoidal profile were achieved. This profile mainly results from the sine interference pattern together with the linear etch rate rise. However, additional to the gratings an overall etching, that is, etching in the fringes minima at $F \sim 0$, was observed especially at higher laser fluences and pulse numbers. Further experiments on overetching of already prepatterned substrates showed that the modulation depth of submicron patterns reduces at overetching with a homogeneous laser beam [65]. The effect was discussed by the better heating of topographic peaks by heat transport from the liquid but the effect seems to be too strong to be completely explained therewith.

Results on high-resolution etching of surface relief line gratings in fused silica down to $104 \mathrm{~nm}$ periods presented in $[45,46]$ show a reduction of the grating depth with the 


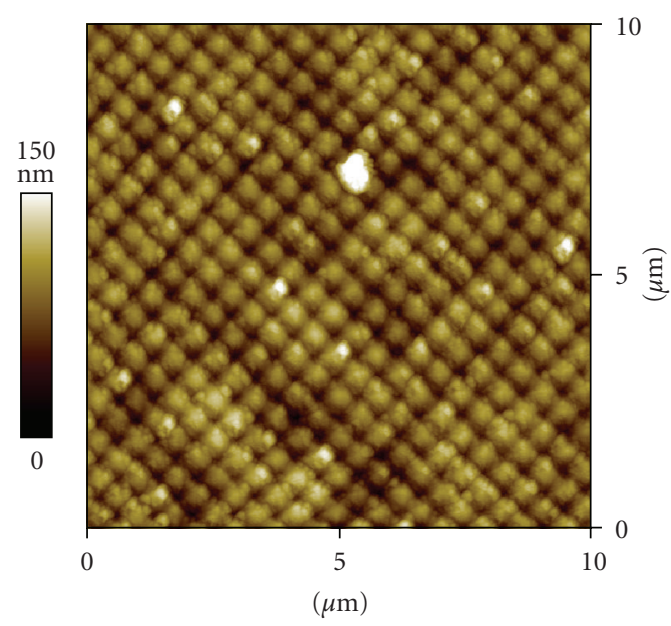

(a)

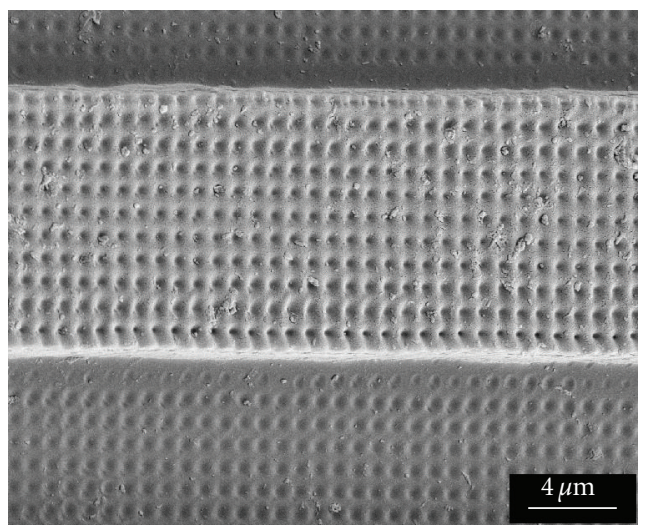

(b)

FIgURE 7: Submicron 2 D gratings with periods $p \sim 550 \mathrm{~nm}$ and $780 \mathrm{~nm}$ etched in fused silica, respectively. (a) Grating obtained by H-LIBWE with a py:acetone solution and (b) grating etched on a prepatterned substrate (line width $\sim 7.5 \mu \mathrm{m}$, prepattern depth $\sim 3 \mu \mathrm{m}$ ) using mercury as absorber.

reduction of the period. This finding can be discussed by the reduction of the modulation of the interface temperature because of the thermal diffusion length of the material given by $L_{T}=2 \cdot \sqrt{D_{T} \cdot t}$ with $D_{T}=k / \rho \cdot c_{p}$. The thermal diffusion lengths $L_{T}$ for fused silica and organic solvents are in the order of 0.18 and $0.09 \mu \mathrm{m}$ for the used 8-nanosecond $266 \mathrm{~nm}$ laser pulses $[45,46]$ and about $0.3 \mu \mathrm{m}$ and $0.15 \mu \mathrm{m}$ for pulse lengths of 25 nanoseconds, respectively. For pattern periods $p$ of similar or smaller than thermal diffusion length (e.g., $\sim 100 \mathrm{~nm}$ ), the heat transport smears interference patterns and only shallow gratings are etched whereas $\sim 0.5 \mu \mathrm{m}$ gratings can be etched proper. This means that highresolution patterns with high modulation depths can be hardly obtained for materials - either the solid and the liquid or both-with high $L_{T}$.

In Figure 7, 2D gratings in FS with periods of about $550 \mathrm{~nm}$ and $780 \mathrm{~nm}$ are shown for $\mathrm{H}-$ and M-LIBWE in comparison. The maximal achieved grating depth of $\sim 160$ to $180 \mathrm{~nm}$ is similar in both cases and is achieved near the threshold of grating etching. However, with M-LIBWE gratings with a depth of $60 \mathrm{~nm}$ can be etched with one laser pulse and can enable submicron patterning "on-the-fly" [48]. The thermal diffusion length of $\sim 0.6 \mu \mathrm{m}$ can result in a strong overetching effect especially at rising laser fluence that can occur than already with the first laser pulse.

\subsubsection{Submicron Grating Etching by Ultrashort Laser Pulses}

To overcome the problem and enable high-quality etching of highly conductive materials by LIBWE, shorter laser pulses must be applied. For 150-picosecond pulse duration, for example, of the fourth harmonic $(266 \mathrm{~nm})$ of an EKSPLA Nd:YAG-laser system, the thermal diffusion length is reduced by a factor greater than 10 and results in thermal diffusion lengths of less than $200 \mathrm{~nm}$ for application-relevant highly conductive materials. For sapphire with a rather high thermal diffusivity $D$ of $1.63 \times 10^{-5} \mathrm{~m}^{2} / \mathrm{s}$, a length of $70 \mathrm{~nm}$ can be calculated.

Surface relief gratings were inscribed in sapphire and in fused silica as well as in different fluorides using H-LIBWE with $266 \mathrm{~nm}, 150$-picosecond laser pulses by applying different interference techniques, for example, phase mask placed in contact to the front surface of the sample. As shown in Figure 8 , the quality of surface relief gratings with an average depth of $\sim 50 \mathrm{~nm}$ etched in sapphire is similar to that achieved for fused silica. However, the threshold laser fluence for etching of gratings is in parts higher for sapphire due to the higher melting and evaporation temperature and due to the, of course, higher thermal conductivity. Details for sapphire grating etching are given in [47].

Therefore, H-LIBWE with ultrashort laser pulses is favourable for etching patterns in several dielectrics with periods shorter than $500 \mathrm{~nm}$ due to the combination of the advantages of ultrashort processing and backside etching [37]. The etching of well-developed $100 \mathrm{~nm}$ patterns with ultrashort laser pulse H-LIBWE in fused silica was shown recently [66].

\section{Mechanism of Laser Etching}

\subsection{Processes at Laser Backside Etching}

The irradiation of the solid-liquid interface by pulsed laser results in a number of processes in both materials, for instance fast heating, transient pressure, shock waves, melting and boiling, bubble formation and collapse, and modification or decomposition of the materials. These processes are linked together in a complex manner and finally cause materials erosion. However, it is generally accepted that laser absorption and heating of the near-interface regions of both materials is the main process for backside etching. 


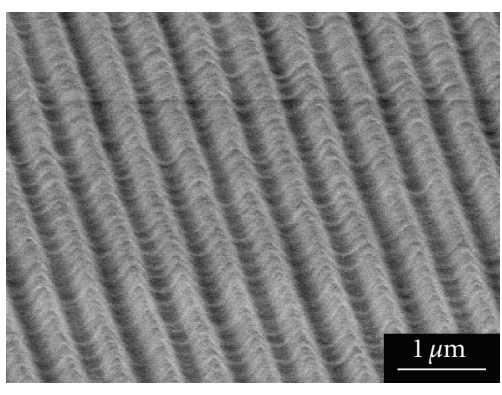

(a)

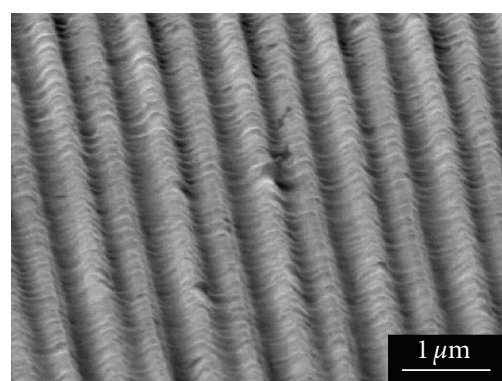

(b)

FIGURE 8: H-LIBWE etched line gratings with a period of $\sim 530 \mathrm{~nm}$ in fused silica (a) and sapphire (b). The SEM images show a similar quality of the inscribed gratings.

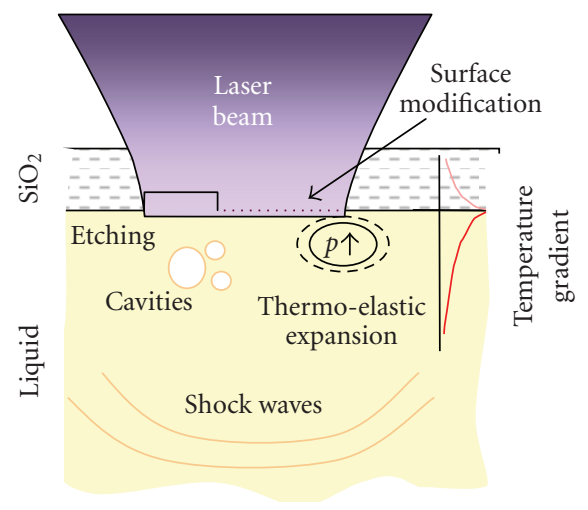

FIGURE 9: Sketch of the main processes at LIBWE. These processes, however, contribute to etching for both LIBWE versions in a different manner.

Figure 9 shows a sketch of the processes in discussion that are involved in the etching mechanism.

In consequence of the laser heating during LIBWE, the softening and the material [23] or phase transitions [32, 40, $50,64]$ are discussed with regards to materials erosion. The softened, destabilized, weakened, or even melted material region near the surface might be expelled by mechanical forces resulting from the fast heating, shockwaves, or bubble formation [23]. The complete modeling of all processes is extremely complex as thermal, mechanical, chemical, and transport processes interacting each other within a very short time have to be considered.

\subsection{Modeling of Thermal Processes at Laser Backside Etching}

Thermal processes are very important for backside etching processes with nanosecond lasers but cannot be neglected for even shorter pulses. A correct solution for the temperature field is very complex because a number of processes are simultaneously coupled to the diffusive heat transfer. Therefore, usually the simple models considering only heat diffusion are used.

In Figure 10, the laser absorption and the temperature profiles are sketched together with the characteristic lengths
$\left(L_{T}, L_{O}=\alpha^{-1}\right)$ for $\mathrm{H}$ - and M-LIBWE. In addition, the laser intensity $I_{L}$ and the laser pulse energy accounting for interface heating $E_{P}^{(I)}$ are shown. From Figure 10, it is clear that the absorbed laser radiation is utilized in a different manner for heating at both LIBWE approaches. Whereas at M-LIBWE $\left(L_{O}^{L} \ll L_{T}^{L}\right)$, the high thermal flux determines the temperature evolution at H-LIBWE $\left(L_{O}^{L} \gg\right.$ $L_{T}^{L}$ ), the laser energy absorption in the liquid mainly affects the temperature distribution and only minor heat energy dissipates from the interface region to the liquid. Therefore, the highest temperature occurs at $\mathrm{H}$ - and M-LIBWE in the liquid and at the interface, respectively. It is also seen, that the temperature profiles at different times $(t 1<t p$ and $t p)$ reflect the different processes.

Although the primary laser-matter interaction upon ultrashort laser irradiation is often different due to the additional processes induced by the short pulses, for example, multiphoton absorption and other nonlinear phenomena, the absorbed energy finally is transformed into heat and can induce similar processes as with nanosecond lasers.

\subsection{Thermal Calculations for LIBWE}

For the rough estimation of the influence of experimental parameters on the etching process, simple thermal models have been developed. As only thermal processes are considered-this does not include the processes of material etching itself-they are only suitable to estimate specific values, for example, the threshold fluence Fth or a critical interface temperature. In such models, regularly constant material parameters and no phase transitions are presumed.

Analytical solutions of the heat equation for surfaces are known for the approximations of fixed coefficients, a constant laser power, and neglecting phase transitions [2, 67]. Further, Carslow/Jaeger gives a solution for heating of an internal interface for interface absorption that can be well applied for M-LIBWE considering the reflectivity and the absorption of the interface [67].

This solution is not valid for H-LIBWE because the absorption coefficient at H-LIBWE is much less, a remarkable interface absorption was measured, and $\left(\alpha^{L}\right)^{-1} \gg L_{T}^{L}$. 


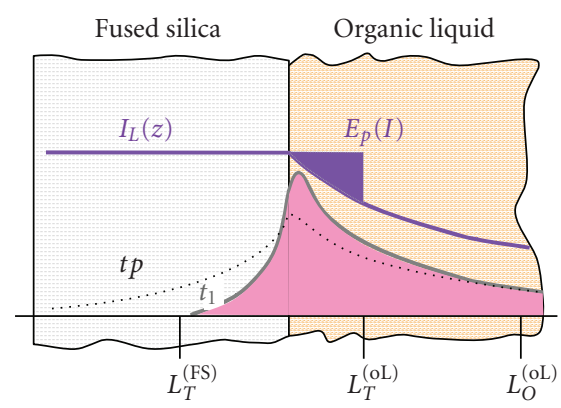

(a)

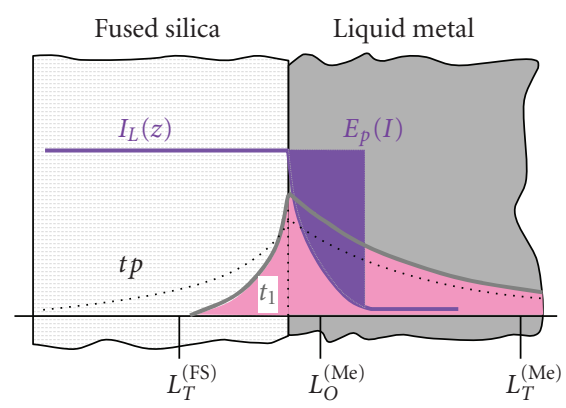

(b)

FIgURE 10: Sketches of the temperature profiles, the laser intensity, and the absorbed laser energy together with the characteristic lengths are shown for $\mathrm{H}$ - and M-LIBWE. $E_{p}$ is the laser pulse energy causing the heating of the interface.

However, an approximation for calculating the interface temperature $T_{I}$ at H-LIBWE is given in [64]. This approximation combines the analytical solution for the interface absorption with a caloric approach accounting for the absorption in the liquid.

An analytical solution for $T_{1}$ for LIBWE for the heating by the laser pulse with a duration $\tau_{p}\left(0 \leq t \leq \tau_{p}\right)$ considering both volume and interface absorption is given in [68] to be

$$
T_{I}=\frac{\sqrt{D_{L}}}{k_{\mathrm{FS}} \cdot \sqrt{D_{L}}+k_{L} \cdot \sqrt{D_{\mathrm{FS}}}} \cdot\left\{I_{V}^{L} \cdot \frac{\alpha_{L}}{4} \cdot L_{T}^{L} \cdot L_{\mathrm{T}}^{\mathrm{FS}}+I_{\mathrm{I}}^{\mathrm{FS}} \cdot \frac{L_{\mathrm{T}}^{\mathrm{FS}}}{\sqrt{\pi}}\right\},
$$

whereas $D, k$, and $L_{T}$ denote the thermal diffusivity, the thermal conductivity, and the heat diffusion length, respectively. $I_{I}$ and $I_{V}$ represent the absorbed laser power at the interface and into the liquid volume and $\alpha$ stands for the absorption coefficient. The superscripts FS and $L$ indicate the fused silica and the liquid that can be either a metal or a hydrocarbon solution, respectively.

The most important outcomes from the models are that higher absorption coefficients of the liquid and/or an elevated absorption of the interface result in higher interface temperatures. A low thermal conductivity and short pulses are additional opportunities to get higher temperatures. Calculated laser-induced temperatures across a solid-liquid interface at H-LIBWE are shown in Figure 11. In the left side (a) shows analytically and numerically calculated temperatures of the fused silica/py:toluene interface with and without interface absorption in comparison and demonstrates the large effect of an additional interface absorption. For example, due to $10 \%$ interface absorption, the temperature rises more then twice and exceeds the boiling temperature of fused silica. Although the temperature gets higher-resulting from the additional interface absorption raise-also for sapphire samples (dashed lines in Figure 11), the effect is much less for sapphire than for fused silica due to the significant higher thermal conductivity $\left(k_{\mathrm{FS}} \sim 1.4\right.$ and $\left.k_{\mathrm{Sp}} \sim 40 \mathrm{~W} / \mathrm{m} \cdot \mathrm{K}\right)$ and does not result in temperatures exceeding the critical temperature for etching, for example, the boiling temperature. Therefore, the low surface quality observed for dielectrics with a high thermal conductivity can be explained by the temperatures field. However, with shorter pulses and a sufficient interface absorption, the melting and boiling of the sapphire surface can be easily achieved as shown in Figure 11(b) in addition.

Combining the experimental found interface absorption values with the experimental applied laser exposure parameters, the interface temperature for H-LIBWE at the multipulse threshold fluence for laser etching of fused silica with a $0.5 \mathrm{M}$ py:toluene absorber was calculated using the mentioned models. Temperatures of more than $4000 \mathrm{~K}$ were calculated at Fth $=0.32 \mathrm{~J} / \mathrm{cm}^{2}$ mainly due to the interface absorption of $\sim 35 \%$ [62]. These extremely high temperatures already at the threshold cause highly dynamic processes of phase transitions and material etching. Furthermore, as the peak power of the (excimer) laser pulse is at the beginning of the pulse and much higher than the average pulse $(F / t p)$ used for the calculations, it is expected that the etching (material removing) occurs within the pulse length. The high temperatures and the expected fast (explosive) processes resemble laser ablation of polymers. However, in the case of H-LIBWE, the ablation-like processes are limited to the near-surface volume that is modified having a very high absorbance. Therefore, the etch rate at H-LIBWE is not predetermined by the thermal diffusion into the fused silica but is given by the amount and the depth of the surface modification.

The comparison of the experimental found etch thresholds at M-LIBWE (Ga, FS, $\lambda=248 \mathrm{~nm}$ ) provides a basically different result [27-29]. Here the first threshold fluence correlates with the melting temperature of fused silica whereas at the second threshold the boiling temperature is exceeded. Some other characteristics of Ga-LIBWE, such as the high etch rates and the smooth, wavelike etched surface with rim-like borders indication melting and resolidification, point at a different etch mechanism. A more evaporationlike mechanism can be suggested from the comparison of the etching efficiency with the only three times larger volumetric energy density for fused silica evaporation.

\subsection{Mechanism of Backside Etching}

The discussion of the experimental results with respect to thermal models shows that basically at least two mechanisms 


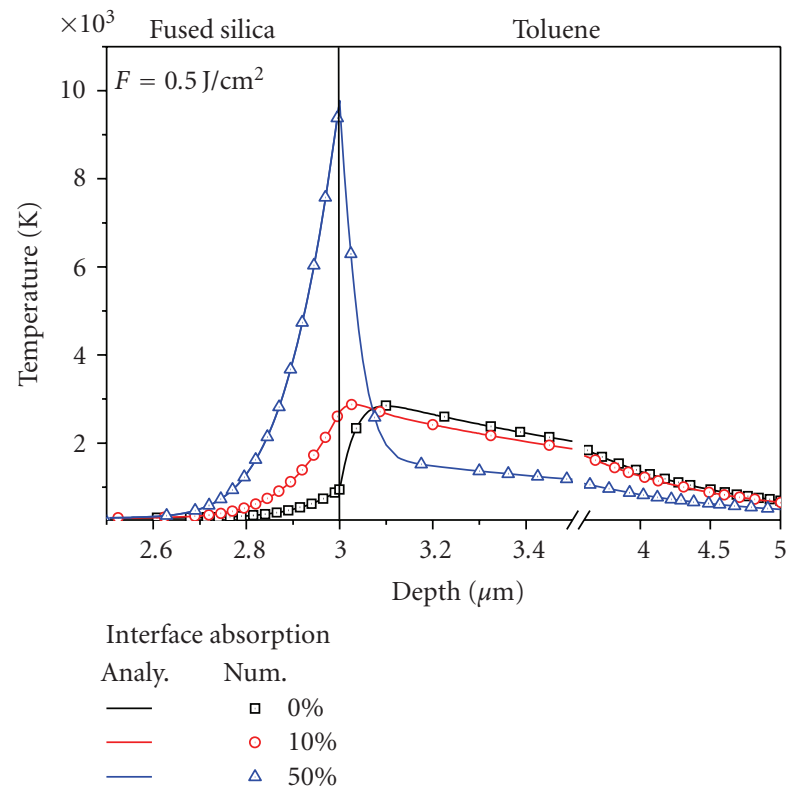

(a)

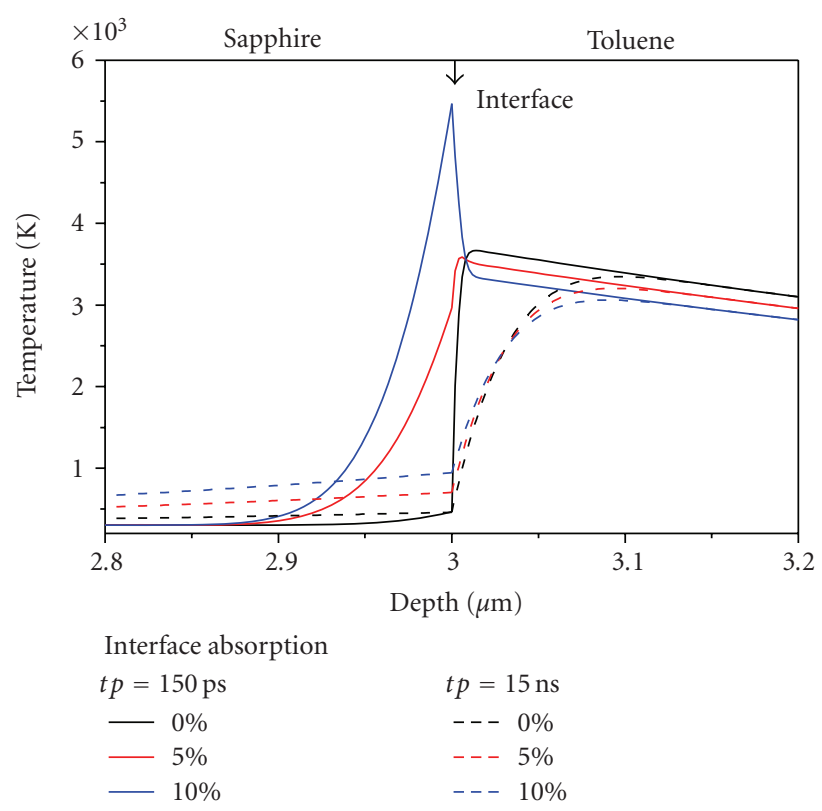

(b)

Figure 11: Calculated temperature profiles across the solid/liquid interface for H-LIBWE (248 nm, py:toluene: (a) analytically and numerically calculated temperatures for fused silica samples of different interface absorption values of 0,10 , and 50\% and (b) sapphire sample for two laser pulse lengths of 15 nanoseconds and 150 picoseconds and different interface absorptions.

of LIBWE have to be distinguished. The first model bases on the heating by a highly absorbing material without significant modification of the solid due to primary or secondary processes at etching, that is, M-LIBWE, and the second mechanism is heavily affected by surface modifications due to secondary processes in combination with fast thermal processes at the solid-liquid interface, that is, H-LIBWE. Additionally, other process characteristics, for example, the confinement of the secondary processes by the absorber, may strongly influence the mechanism at all as the comparison of backside ablation in water/air confinement has been shown [69]. Therefore, the interaction strength of secondary processes, for example, shock waves, liquid decomposition and material deposition, laser-induced plasma and rapid melting and resolidification, with the solid surface depends also on material characteristics of the absorber (liquid), the possible and most likely paths of excitation and relaxation, and the interaction time; all effects are influenced by the confinement.

The sketch shown in Figure 12 gives an overview on the mechanism and the near-surface processes essential for hydrocarbon laser-induced backside wet etching. Here the main focus is on the surface whereas the other processes already mentioned and shown in the figure are also going on.

At the first laser pulse with a fluence above the etching threshold $\mathrm{Fth}(\infty)$, the laser radiation is absorbed by the organic liquid and heats it up to temperatures below the melting temperature of the solid. However, the organic solution vaporizes and decomposes due to photothermal and photochemical processes so that the formation of bubbles is forced. Due to heating, decomposition, and bubble formation, the surface is modified increasingly with the pulse number by liquid decomposition products, for example, carbon, resulting in a thin highly absorbing film on top of the solid. Due to this, the solid surface temperature increases with the pulse number and exceeds a critical temperature $T_{c}$ at $N_{\text {inc }}$. At this state, the transparent solid material is etched [58]. However, as has been shown, the temperatures that can be achieved at etching are very high so that ablationlike processes occur at the solid surface that is confined by the liquid. This confinement may enhance the interaction of secondary processes with the solid and results in a modified near-surface region of the solid with a high absorption as shown in the figure. This modified near-surface solid region highly absorbs the UV laser radiation and results in an efficient interface heating. At this point the process of surface modification is self-maintaining but needs the absorbing liquid. This general view is more or less changed by the experimental conditions used.

With this extended model considering process-induced surface modifications, some experimental findings can be explained. So the threshold fluence correlates at different UV wavelengths not with the absorption coefficients of the liquid but with the measured absorption in the modified layer according to the extended mechanism. Probably in the same manner the neglectable change of the etching threshold with the absorption coefficient of the liquid absorber (due to dye, e.g., pyrene, admixture) can be explained because the threshold is mainly given by the enhancement of the absorption by carbon-film surface modification from decomposition processes that also appear without dyes. 


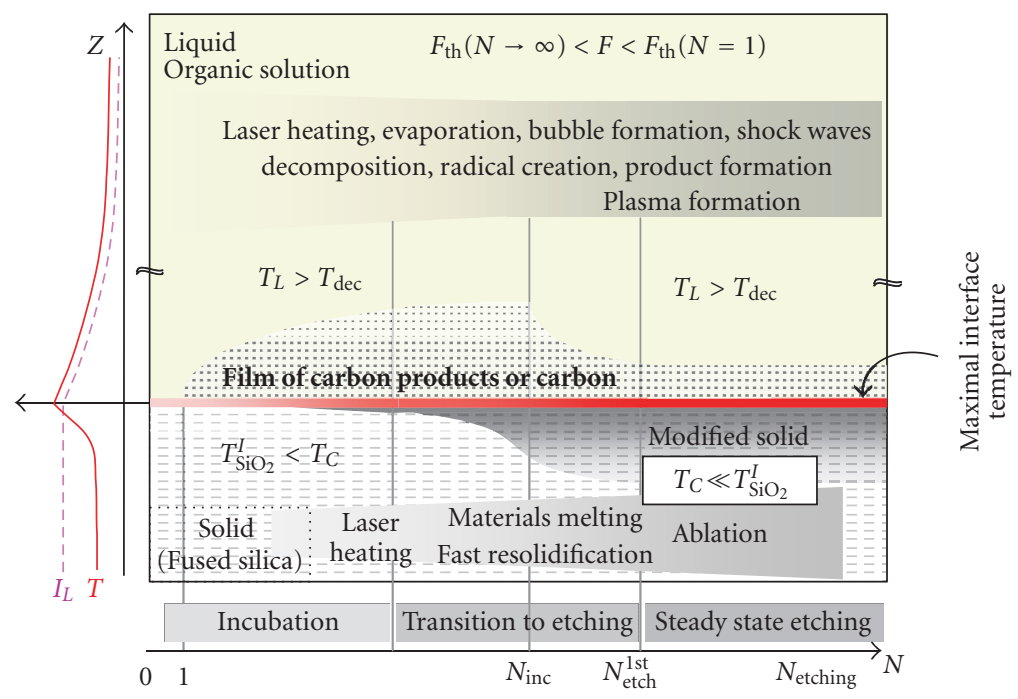

FIGURE 12: Schematic sketch of the processes at H-LIBWE. In particular, the changes of the surface modification at prolonged laser irradiation are shown and probably explain the observed incubation effects at H-LIBWE. The gray level is a measure of the strength.

The concrete processes of solid modifications depend, of course, on the composition of the organic liquid absorber whereby the different thresholds can be explained, for instance.

\section{Summary}

High quality etching of transparent materials with pulsed laser radiation can be achieved by two different LIBWE approaches using hydrocarbon or metallic liquid absorbers. The processing parameters, the achievable patterns, and the surface morphology depend on the used absorber. Hence, the processing parameters such as wavelength, liquid absorber, laser fluence, and so forth must be well chosen with respect to the solid material and the processing goal. The thermal modeling of H-LIBWE shows that the highly absorbing surface modification will cause extreme high temperatures of the modified solid surface that probably results in ablation-like material erosion processes. Submicron gratings can be etched by LIBWE not only in thermal low conducting materials such as fused silica using organic or metallic absorbers but also in crystalline materials, for example, sapphire, applying well-chosen parameters to meet the preconditions for surface modification and high-modulation surface heating of the transparent sample.

\section{Acknowledgments}

The authors gratefully acknowledge the financial support of the Deutsche Forschungsgemeinschaft (DFG ZI 660/5-2 and DFG ZI660/3), the EU within the Research Infrastructures activities of FP6 (Project: Laserlab-Europe; Contract no. RII3-CT-2003-506350), and the Deutscher Akademischer Austausch Dienst (DAAD D/06/05877). The authors are deeply indebted to S. Pissadakis and B. Hopp for their help and interest in the work and the fruitful discussions. The authors acknowledge M. Erhardt, Ph. Hadrava, D. Hirsch, R. Fechner, P. Hertel, and E. Salamatin for their diligence in contributing to the experimental work and for the careful reading of the manuscript.

\section{References}

[1] S. Sinzinger and J. Jahns, Microoptics, Wiley-VCH, Berlin, Germany, 2003.

[2] D. Bäuerle, Laser Processing and Chemistry, Springer, Berlin, Germany, 3rd edition, 2000.

[3] B. Lan, M.-H. Hong, K.-D. Ye, Z.-B. Wang, S.-X. Cheng, and T.-C. Chong, "Laser precision engineering of glass substrates," Japanese Journal of Applied Physics, vol. 43, no. 10, pp. 71027106, 2004.

[4] J. Ihlemann and B. Wolff-Rottke, "Excimer laser micro machining of inorganic dielectrics," Applied Surface Science, vol. 106, pp. 282-286, 1996.

[5] J. Ihlemann, B. Wolff, and P. Simon, "Nanosecond and femtosecond excimer laser ablation of fused silica," Applied Physics A, vol. 54, no. 4, pp. 363-368, 1992.

[6] M. Lenzner, J. Krüger, W. Kautek, and F. Krausz, "Incubation of laser ablation in fused silica with 5-fs pulses," Applied Physics A, vol. 69, no. 4, pp. 465-466, 1999.

[7] W. Kautek, J. Krüger, M. Lenzner, S. Sartania, C. Spielmann, and F. Krausz, "Laser ablation of dielectrics with pulse durations between 20 fs and 3 ps," Applied Physics Letters, vol. 69, no. 21, pp. 3146-3148, 1996.

[8] D. Schäfer, J. Ihlemann, G. Marowsky, and P. R. Herman, "F2laser ablation patterning of dielectric layers," Applied Physics A, vol. 72, no. 3, pp. 377-379, 2001.

[9] P. R. Herman, R. S. Marjoribanks, A. Oettl, K. Chen, I. Konovalov, and S. Ness, "Laser shaping of photonic materials: deep-ultraviolet and ultrafast lasers," Applied Surface Science, vol. 154, pp. 577-586, 2000.

[10] K. Sugioka, S. Wada, H. Tashiro, K. Toyoda, Y. Ohnuma, and A. Nakamura, "Multiwavelength excitation by vacuumultraviolet beams coupled with fourth harmonics of a $Q$ switched Nd:YAG laser for high-quality ablation of fused 
quartz," Applied Physics Letters, vol. 67, no. 19, pp. 2789-2791, 1995.

[11] K. Sugioka, J. Zhang, S. Wada, H. Tashiro, and K. Toyoda, "Novel ablation of wide band-gap materials by multiwavelength excitation using a VUV-UV laser system," Nanotechnology, vol. 9, no. 2, pp. 99-103, 1998.

[12] J. Zhang, K. Sugioka, and K. Midorikawa, "High-quality and high-efficiency machining of glass materials by laser-induced plasma-assisted ablation using conventional nanosecond UV, visible, and infrared lasers," Applied Physics A, vol. 69, no. 7, pp. S879-S882, 1999.

[13] J. Zhang, K. Sugioka, and K. Midorikawa, "Laser-induced plasma-assisted ablation of fused quartz using the fourth harmonic of a $\mathrm{Nd}^{+}$:YAG laser," Applied Physics A, vol. 67, no. 5, pp. 545-549, 1998.

[14] J. Zhang, K. Sugioka, and K. Midorikawa, "Direct fabrication of microgratings in fused quartz by laser-induced plasmaassisted ablation with a KrF excimer laser," Optics Letters, vol. 23, no. 18, pp. 1486-1488, 1998.

[15] K. Sugioka, K. Obata, K. Midorikawa, et al., "Advanced materials processing based on interaction of laser beam and a medium," Journal of Photochemistry and Photobiology A, vol. 158, no. 2-3, pp. 171-178, 2003.

[16] K. Sugioka, K. Obata, M. H. Hong, et al., "Hybrid laser processing for microfabrication of glass," Applied Physics A, vol. 77, no. 2, pp. 251-257, 2003.

[17] R. Böhme and K. Zimmer, "Low roughness laser etching of fused silica using an adsorbed layer," Applied Surface Science, vol. 239, no. 1, pp. 109-116, 2004.

[18] R. Böhme, K. Zimmer, D. Ruthe, and B. Rauschenbach, "Backside etching at the interface to diluted medium with nanometer etch rates," Journal of Laser Micro/Nanoengineering, vol. 1, no. 3, pp. 190-194, 2006.

[19] K. Zimmer, R. Böhme, and B. Rauschenbach, "Laser etching of fused silica using an adsorbed toluene layer," Applied Physics A, vol. 79, no. 8, pp. 1883-1885, 2004.

[20] K. Zimmer, R. Böhme, and B. Rauschenbach, "Adsorbed layer etching of fused silica by excimer laser with nanometer depth precision," Microelectronic Engineering, vol. 78-79, no. 1-4, pp. 324-330, 2005.

[21] T. Smausz, T. Csizmadia, N. Kresz, C. Vass, Z. Márton, and B. Hopp, "Influence on the laser induced backside dry etching of thickness and material of the absorber, laser spot size and multipulse irradiation," Applied Surface Science, vol. 254, no. 4, pp. 1091-1095, 2007.

[22] J. Wang, H. Niino, and A. Yabe, "Micromachining of quartz crystal with excimer lasers by laser-induced backside wet etching," Applied Physics A, vol. 69, no. 7, pp. S271-S273, 1999.

[23] J. Wang, H. Niino, and A. Yabe, "One-step microfabrication of fused silica by laser ablation of an organic solution," Applied Physics A, vol. 68, no. 1, pp. 111-113, 1999.

[24] J. Wang, H. Niino, and A. Yabe, "Micromachining of transparent materials with super-heated liquid generated by multiphotonic absorption of organic molecule," Applied Surface Science, vol. 154, pp. 571-576, 2000.

[25] S. I. Dolgaev, A. A. Lyalin, A. V. Simakin, V. V. Voronov, and G. A. Shafeev, "Fast etching and metallization of via-holes in sapphire with the help of radiation by a copper vapor laser," Applied Surface Science, vol. 109-110, pp. 201-205, 1997.

[26] S. I. Dolgaev, A. A. Lyalin, A. V. Simakin, and G. A. Shafeev, "Fast etching of sapphire by a visible range quasi-cw laser radiation," Applied Surface Science, vol. 96-98, pp. 491-495, 1996.
[27] K. Zimmer, R. Böhme, D. Ruthe, and B. Rauschenbach, "Backside laser etching of fused silica using liquid gallium," Applied Physics A, vol. 84, no. 4, pp. 455-458, 2006.

[28] K. Zimmer, R. Böhme, and B. Rauschenbach, "Using IR laser radiation for backside etching of fused silica," Applied Physics A, vol. 86, no. 3, pp. 409-414, 2007.

[29] K. Zimmer, R. Böhme, D. Hirsch, and B. Rauschenbach, "Backside etching of fused silica with UV laser pulses using mercury," Journal of Physics D, vol. 39, no. 21, pp. 4651-4655, 2006.

[30] J.-Y. Cheng, M.-H. Yen, C.-W. Wei, Y.-C. Chuang, and T.-H. Young, "Crack-free direct-writing on glass using a low-power UV laser in the manufacture of a microfluidic chip," Journal of Micromechanics and Microengineering, vol. 15, no. 6, pp. 11471156, 2005.

[31] H. Niino, Y. Kawaguchi, T. Sato, A. Narazaki, T. Gumpenberger, and R. Kurosaki, "Surface micro-structuring of silica glass by laser-induced backside wet etching with nspulsed laser at high repetition rate," Journal of Laser Micro/Nanoengineering, vol. 1, no. 1, pp. 39-43, 2006.

[32] C. Vass, T. Smausz, and B. Hopp, "Wet etching of fused silica: a multiplex study," Journal of Physics D, vol. 37, no. 17, pp. 2449-2454, 2004.

[33] X. Ding, Y. Kawaguchi, H. Niino, and A. Yabe, "Laser-induced high-quality etching of fused silica using a novel aqueous medium," Applied Physics A, vol. 75, no. 6, pp. 641-645, 2002.

[34] X. Ding, Y. Yasui, Y. Kawaguchi, H. Niino, and A. Yabe, "Laserinduced back-side wet etching of fused silica with an aqueous solution containing organic molecules," Applied Physics A, vol. 75, no. 3, pp. 437-440, 2002.

[35] R. Böhme and K. Zimmer, "Indirect laser etching of fused silica: towards high etching rate processing," Applied Surface Science, vol. 253, no. 19, pp. 8091-8096, 2007.

[36] C. Vass, D. Sebök, and B. Hopp, "Comparing study of subpicosecond and nanosecond wet etching of fused silica," Applied Surface Science, vol. 252, no. 13, pp. 4768-4772, 2006.

[37] R. Böhme, S. Pissadakis, M. Ehrhardt, D. Ruthe, and K. Zimmer, "Ultra-short laser processing of transparent material at the interface to liquid," Journal of Physics D, vol. 39, no. 7, pp. 1398-1404, 2006.

[38] R. Böhme, D. Spemann, and K. Zimmer, "Surface characterization of backside-etched transparent dielectrics," Thin Solid Films, vol. 453-454, pp. 127-132, 2004.

[39] G. Kopitkovas, T. Lippert, C. David, S. Canulescu, A. Wokaun, and J. Gobrecht, "Fabrication of beam homogenizers in quartz by laser micromachining," Journal of Photochemistry and Photobiology A, vol. 166, no. 1-3, pp. 135-140, 2004.

[40] C. Vass, B. Hopp, T. Smausz, and F. Ignácz, "Experiments and numerical calculations for the interpretation of the backside wet etching of fused silica," Thin Solid Films, vol. 453-454, pp. 121-126, 2004.

[41] K. Zimmer, A. Braun, and R. Böhme, "Etching of fused silica and glass with excimer laser at $351 \mathrm{~nm}$," Applied Surface Science, vol. 208-209, no. 1, pp. 199-204, 2003.

[42] G. Kopitkovas, T. Lippert, C. David, A. Wokaun, and J. Gobrecht, "Surface micromachining of UV transparent materials," Thin Solid Films, vol. 453-454, pp. 31-35, 2004.

[43] X. Ding, T. Sato, Y. Kawaguchi, and H. Niino, "Laser-induced backside wet etching of sapphire," Japanese Journal of Applied Physics, vol. 42, no. 2B, pp. L176-L178, 2003.

[44] K. Zimmer, R. Böhme, A. Braun, B. Rauschenbach, and F. Bigl, "Excimer laser-induced etching of sub-micron surface relief 
gratings in fused silica using phase grating projection," Applied Physics A, vol. 74, no. 4, pp. 453-456, 2002.

[45] C. Vass, K. Osvay, and B. Hopp, "Fabrication of $150 \mathrm{~nm}$ period grating in fused silica by two-beam interferometric laser induced backside wet etching method," Optics Express, vol. 14, no. 18, pp. 8354-8359, 2006.

[46] C. Vass, K. Osvay, B. Hopp, and Z. Bor, " $104 \mathrm{~nm}$ period grating fabrication in fused silica by immersion two-beam interferometric laser induced backside wet etching technique," Applied Physics A, vol. 87, no. 4, pp. 611-613, 2007.

[47] S. Pissadakis, R. Böhme, and K. Zimmer, "Sub-micron periodic structuring of sapphire by laser induced backside wet etching technique," Optics Express, vol. 15, no. 4, pp. 1428 1433, 2007.

[48] R. Böhme and K. Zimmer, "Laser-induced writing of submicron surface relief gratings in fused silica on the fly," Journal of Laser Micro/Nanoengineering, vol. 2, no. 3, pp. 178-182, 2007.

[49] G. Kopitkovas, T. Lippert, N. Murazawa, et al., "Laser processing of micro-optical components in quartz," Applied Surface Science, vol. 254, no. 4, pp. 1073-1078, 2007.

[50] G. Kopitkovas, T. Lippert, C. David, A. Wokaun, and J. Gobrecht, "Fabrication of micro-optical elements in quartz by laser induced backside wet etching," Microelectronic Engineering, vol. 67-68, pp. 438-444, 2003.

[51] K. Zimmer and R. Böhme, "Precise etching of fused silica for refractive and diffractive micro-optical applications," Optics and Lasers in Engineering, vol. 43, no. 12, pp. 1349-1360, 2005.

[52] K. Zimmer and R. Böhme, "Precise etching of fused silica for micro-optical applications," Applied Surface Science, vol. 243, no. 1-4, pp. 415-420, 2005.

[53] R. Böhme, K. Zimmer, and B. Rauschenbach, "Direct laser etching of transparent materials: writing of high-quality micro- and submicron-structures enables surface features for optical applications," in Proceedings of the 7th International Conference on Correlation Optics, vol. 6254 of Proceedings of SPIE, pp. 1-9, Chernivtsi, Ukraine, September 2006.

[54] T. Gumpenberger, T. Sato, R. Kurosaki, A. Narazaki, Y. Kawaguchi, and H. Niino, "Fabrication of a novel microfluidic device incorporating 2-D array of microbeads," Chemistry Letters, vol. 35, no. 2, pp. 218-219, 2006.

[55] K. Zimmer and A. Braun, "Excimer laser machining for 3Dsurface structuring," in Photo-Excited Processes, Diagnostics and Applications, A. Peled, Ed., pp. 301-335, Kluwer Academic Publishers, Boston, Mass, USA, 2003.

[56] K. Zimmer, R. Böhme, D. Hirsch, and B. Rauschenbach, "Backside etching of fused silica with UV laser pulses using mercury," Journal of Physics D, vol. 39, no. 21, pp. 4651-4655, 2006.

[57] Y. Tsuboi, K. Hatanaka, H. Fukumura, and H. Masuhara, "The $248 \mathrm{~nm}$ excimer laser ablation of liquid benzene derivatives: a relation between ablation threshold and molecular photochemical reactivity," Journal of Physical Chemistry, vol. 98, no. 44, pp. 11237-11241, 1994.

[58] R. Böhme, Laser-induced backside wet etching of glasses and crystals, Ph.D. thesis, Zentrum für Ingenieurwissenschaften, Martin-Luther-Universität, Halle-Wittenberg, Halle, Germany, 2007.

[59] R. Böhme and K. Zimmer, "Effects of halogenated organic solvents on laser-induced backside wet etching of fused silica," Applied Physics A, vol. 83, no. 1, pp. 9-12, 2006.

[60] K. Hatanaka, M. Kawao, Y. Tsuboi, H. Fukumura, and H. Masuhara, "Switching from photochemical to photothermal mechanism in laser ablation of benzene solutions," Journal of Applied Physics, vol. 82, no. 11, pp. 5799-5806, 1997.

[61] Y. Tsuboi, K. Hatanaka, H. Fukumura, and H. Masuhara, "The 248-nm excimer-laser-ablation mechanism of liquid benzene derivatives: photochemical formation of benzyl radical leads to ablation," Journal of Physical Chemistry A, vol. 102, no. 10, pp. 1661-1665, 1998.

[62] R. Böhme, Laser-Based Etching Technique for Micro/Nano Patterning of Transparent Materials, VDM, Saarbrücken, Germany, 2007.

[63] R. Böhme, A. Braun, and K. Zimmer, "Backside etching of UV-transparent materials at the interface to liquids," Applied Surface Science, vol. 186, no. 1-4, pp. 276-281, 2002.

[64] K. Zimmer, R. Böhme, D. Ruthe, and B. Rauschenbach, "The influence of laser-induced surface modifications on the backside etching process," Applied Surface Science, vol. 253, no. 15, pp. 6588-6594, 2007.

[65] R. Böhme, J. Zajadacz, K. Zimmer, and B. Rauschenbach, "Topography and roughness evolution of microstructured surfaces at laser-induced backside wet etching," Applied Physics A, vol. 80, no. 2, pp. 433-438, 2005.

[66] R. Böhme, C. Vass, B. Hopp, and K. Zimmer, "Sub-wavelength ripples in fused silica after the irradiation the solid/liquid interface with ultrashort laser pulses," to appear in Nanotechnologies.

[67] H. S. Carslaw and J. C. Jaeger, Conduction of Heat in Solids, Oxford University Press, Oxford, UK, 1959.

[68] K. Zimmer, "Analytical solution of the laser-induced temperature distribution across internal material interfaces," International Journal of Heat and Mass Transfer. In press.

[69] R. Böhme, K. Zimmer, and B. Rauschenbach, "Laser backside etching of fused silica due to carbon layer ablation," Applied Physics A, vol. 82, no. 2, pp. 325-328, 2006. 